\title{
Isolation and characterization of renin-like aspartic-proteases from Echis ocellatus
} venom

\author{
M.C. Wilkinson ${ }^{\text {a },}$, D.J.H. Nightingale ${ }^{a}$, R.A. Harrison ${ }^{b}$, S.C. Wagstaff ${ }^{b}$ \\ a Institute of Integrative Biology, University of Liverpool, Liverpool, L69 7ZB 4, UK \\ ${ }^{\mathrm{b}}$ Liverpool School of Tropical Medicine, Liverpool, L3 5QA, UK
}

A R T I C L E I N F O

\section{Article history:}

Received 28 June 2017

Accepted 6 July 2017

Available online xxx

Keywords:

Aspartic protease

Echis ocellatus

Hypertension

Renin

\begin{abstract}
A B S T R A C T
Three aspartic proteases (SVAPs) have been isolated from venom of the saw-scaled viper, Echis ocellatus. In confirmation of prior transcriptomic predictions, all three forms match to sequences of either of the two SVAP transcripts (EOC00051 and EOC00123), have a molecular weight of $42 \mathrm{kDa}$ and possess a single N-glycan. The SVAPs act in a renin-like manner, specifically cleaving human and porcine angiotensinogen into angiotensin-1 and possess no general protease activity. Their activity is completely inhibited by the aspartyl protease inhibitor Pepstatin A.
\end{abstract}

In a previous study we presented evidence for the existence of renin-like aspartic proteases in the venom gland transcriptome of the West African (Nigeria) saw-scaled viper, Echis ocellatus (Wagstaff and Harrison, 2006). This was the first report of this protein group being expressed in snakes. Full-length sequences of two aspartic proteases were determined, EOC00051 [AM180265] and EOC00123 [AM180266], and in both the presence of a signal peptide sequence was identified. This, and the absence of lysosomal targeting sequences, strongly indicated that these proteases would be secreted components of venom. Analysis of the two sequences also revealed $>50 \%$ overall sequence identity with human (P00797) and mouse (P06281) renin-1, including conserved cysteine patterns and catalytic residues. Our evidence thus suggested that aspartic proteases with renin-like activity may be $E$. ocellatus venom components and therefore function as a new class of venom toxins. Here we outline the results of further studies carried out to isolate these proteases from the venom and confirm their renin-like activity.

\section{Confirmation of aspartic proteases in venom of $E$. ocellatus}

To detect aspartic proteases in venom, we first generated a polyclonal antibody specific to E. ocellatus venom aspartic proteases (SVAP) by immunizing a rabbit with a multi-antigenic RYDSSESSTYKPKGT peptide immunogen: this peptide corresponds to residues $122-136$ of both translated transcripts, EOC00051 and EOC00123. This antibody reacted with a band at approximately

\footnotetext{
* Corresponding author.

Email address: mwilk@liv.ac.uk (M.C. Wilkinson)
}

$42 \mathrm{kDa}$ in immunoblots of E. ocellatus (i) whole venom and (ii) gel filtration chromatography (GFC)-separated venom proteins (data not shown). This band was evident on Coomassie blue-stained venom SDS-PAGE gels (at a level estimated to be equivalent to around $2 \%$ of total venom protein as predicted by our transcriptional data) and was excised and subjected to in-gel trypsin digestion for peptide mass analysis. Nine of the resultant peptides matched sequences of the original SVAP transcripts (Table 1, column WV). In addition to these matching peptide masses, we obtained the sequence of the tryptic peptide GFFSQDIVR (res 149-157) by ES-MS-MS. This venom proteomic data clearly confirmed that the protein[s] contained in this band matched to the transcripts EOC00123 and EOC00051 in the E. ocellatus transcriptome.

\section{Isolation of three isoforms of venom aspartic proteases}

Following identification of the $42 \mathrm{kDa}$ band as the putative SVAP, we set out to isolate sufficient amounts of the purified protein for function analysis. Fifty $\mathrm{mg}$ of E. ocellatus (Nigeria) venom in $5 \mathrm{~mL}$ PBS (50 mM sodium phosphate, $150 \mathrm{mM} \mathrm{NaCl}, \mathrm{pH} 7.2$ ) was subjected to gel filtration chromatography (GFC) on a $90 \mathrm{~cm} \times 2.6 \mathrm{~cm}$ Superdex 200 column. Elution was carried out at $2.0 \mathrm{~mL} / \mathrm{min}$. The putative SVAP ( $42 \mathrm{kDa}$ band on SDS-PAGE) was partially purified by this chromatographic procedure, eluting just after the $65 \mathrm{kDa}$ venom metalloproteases. Further purification was carried out using anion exchange chromatography on a $1 \mathrm{~mL}$ Mono Q column (GE Healthcare), eluting with a $0-0.3 \mathrm{M}$ gradient of $\mathrm{NaCl}$ in $50 \mathrm{mM}$ Tris-Cl, $\mathrm{pH}$ 7.8. Multiple peaks were routinely detected for the SVAPs using this separation method, providing evidence for at least three main isoforms, which we designated SVAP B1, SVAP A1 
Table 1

Peptide mass matches from the MALDI-TOF spectrum of peptides obtained through in-gel digestion of $42 \mathrm{kDa}$ bands from SDS-PAGE gels of whole venom [WV], or following digestion of isolated SVAP isoforms AI, B1 and B2.

\begin{tabular}{llllll}
\hline Mass (Da) & Peptide & WV & A1 & B1 & B2 \\
\hline 3309.6 & HSESNTGGEIILGGSDPSHYTGDFHYVSTSR & Y & & & \\
3190.6 & VVFDTGSSNLWVPSHQCSPLYSACVSHNR & Y & & Y & Y \\
2274.3 & ITLTYGQGYIEGFLSQDIVR & Y & & Y & Y \\
2032.1 & DTQYYGEISIGTPAQIFK & Y & & & \\
1819.1 & QAIGGVIPVFDNIMSEK & Y & & & \\
1664.0 & VVFDTGSSNLWVPS & Y & Y & & \\
1562.9 & VLSENVFSVYYSR & Y & Y & Y & Y \\
1315.8 & GPLWLLGATFIK & Y & & Y & Y \\
1068.5 & GFFQDIVR & Y & Y & & \\
\hline
\end{tabular}

Sequences were obtained from the theoretical digest tool (http://ca.expasy.org/tools/ peptide-mass.html). Double-underlined sequences $(=)$ are unique to EOC00051, single-underlined sequences ( - ) are unique to EOC00123 and sequences possessing no underlining are common to both isoforms.

and SVAP B2 (in order of elution from Mono Q) based on their sequence matches to the transcripts $(E O C 00051=$ form A; EOC00123 = form B, see Table 1). A third chromatography step was carried out in order to acquire highly purified samples of SVAP A1,
SVAP B1 and SVAP B2 isoforms. Mono Q fractions containing both SVAP A1 and SVAP B1 were loaded onto a $1 \mathrm{~mL}$ Phenyl Superose hydrophobic interaction chromatography (HIC) column (GE Healthcare) equilibrated in $50 \mathrm{mM}$ TrisCl, $3 \mathrm{M} \mathrm{NaCl}, \mathrm{pH} 7.8$ and eluted with a gradient of decreasing $\mathrm{NaCl}$ concentration. The two isoforms $\mathrm{A} 1$ and B1 were fully separated from each other using HIC. SVAP B2 was also further purified on HIC during which it chromatographed as a single peak. SDS-PAGE analysis of three purified isoforms (Fig. 1A) reveals that SVAP A1 has a slightly lower molecular mass than that of SVAP B1 and SVAP B2. Multiple bands can be seen for each isoform suggesting further structural variation within each isoform. This variation may be due to the presence of glycoforms: all three isoforms ran with higher mobility on SDS-PAGE following overnight treatment with PNGase F to remove N-glycans; the apparent mwt decreased by Ca. $4 \mathrm{kDa}$, suggesting a single $\mathrm{N}$-glycan (data not shown). Both transcript sequences have a single potential N-glycosylation site at residue 64. Using trypsin digestion and mass spectrometry [MS] analysis to isolate and identify peptides unique to either of the two transcripts, we were able to confirm that SVAP A1 is derived from EOC00051, whereas SVAP B1 and SVAP B2 are derived from transcript EOC00123 (Table 1).
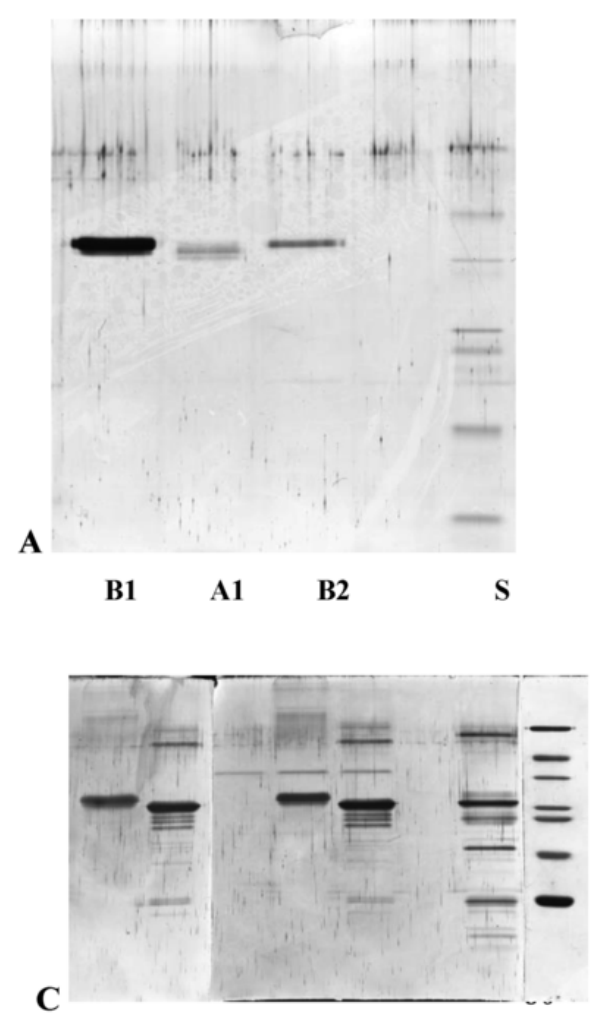

$\begin{array}{lllllll}1 & 2 & 3 & 4 & 5 & 6 & \mathrm{~S}\end{array}$

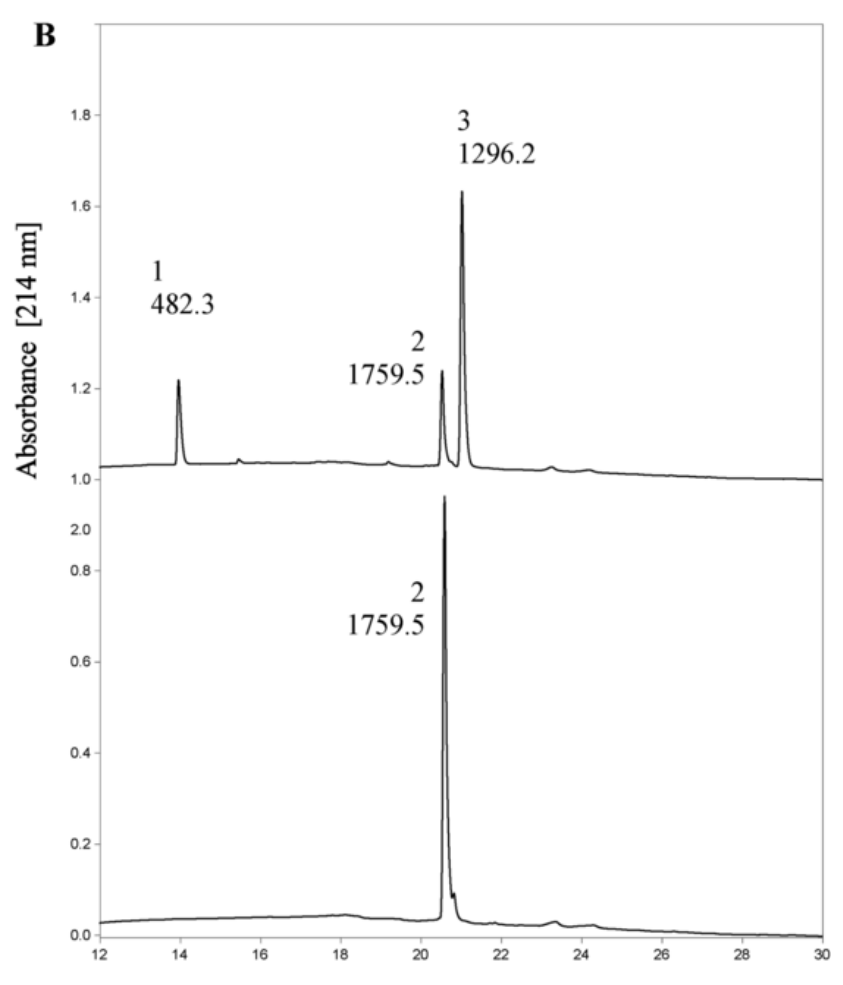

Residence time [mins]

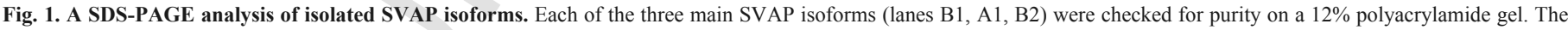

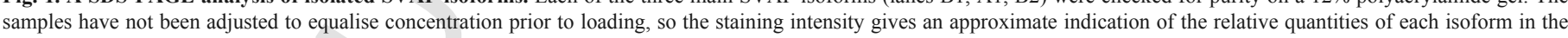

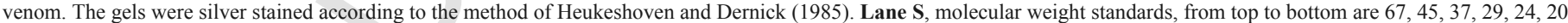

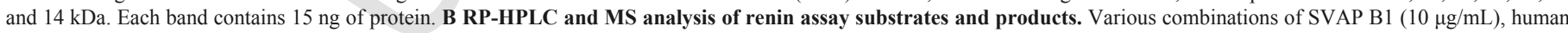

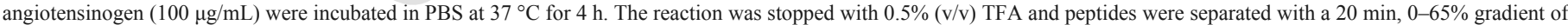

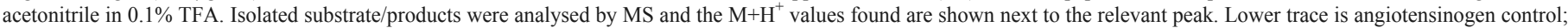

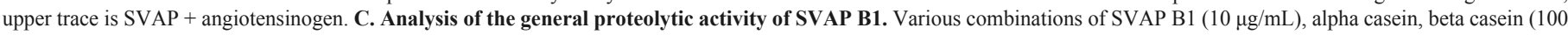

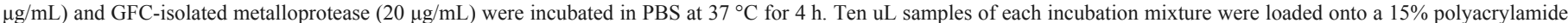

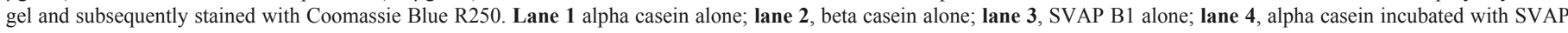

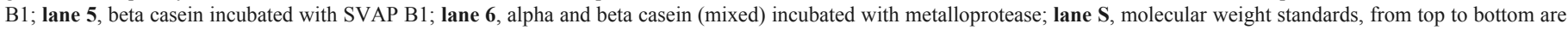
$67,45,37,29,24,20$ and $14 \mathrm{kDa}$. 


\section{Confirmation of the renin-like function of venom aspartic proteases}

Since SVAP B1 was routinely found to be the most abundant of the three isoforms in E. ocellatus venom, this protein was used to develop the assay for renin-like activity. The assay incubation mixture contained the tetradecapeptide substrate, human angiotensinogen (DRVYIHPFHLVIHN; at a concentration of $100 \mu \mathrm{g} / \mathrm{mL}$ in PBS) with SVAP B1 at $10 \mu \mathrm{g} / \mathrm{mL}$ and was incubated at $37^{\circ} \mathrm{C}$ for $1-20 \mathrm{~h}$. MS was used to analyse the decapeptide (angiotensin-1, M+H+ 1296.0) and tetrapeptide (VIHN, $\mathrm{M}+\mathrm{H}+482.3$ ) products arising from renin like cleavage between residues 10 and 11 . Although direct analysis for angiotensin-1 on MALDI-TOF provided a rapid measure of renin-like activity, a C18 reverse-phase HPLC separation of substrate and products along with subsequent MALDI-MS or ESI-MS analysis of the peaks allowed for a semi-quantitative measure of activity and a more complete analysis of the specificity of SVAP B1 towards the substrate (example, Fig. 1B). The results of all these analyses confirmed the renin-like specificity of SVAP B1. Thus, we identified (by both mass and sequence) the presence of the tetrapeptide product VIHN which hadn't been detectable using MALDI-MS. The presence of this peptide product provided evidence for a specific cleavage event rather than the possibility of a carboxypeptidase-like activity of the SVAP being responsible for the generation of angiotensin-1 from angiotensinogen. Under the conditions used here, conversion was typically $70-80 \%$ complete within $3 \mathrm{~h}$. This activity was almost completely inhibited by the aspartyl protease inhibitor, Pepstatin A, at a concentration of $10 \mu \mathrm{M}$, but was not inhibited by the serine or metalloproteinase inhibitors, PMSF or EDTA. All three isoforms were shown to be active towards human angiotensinogen and to be inhibited by $10 \mu \mathrm{M}$ Pepstatin A. These analyses were successfully repeated using porcine angiotensinogen (DRVYIHPFHLLVYS).

Using a lower purity SVAP fraction from the anion exchange separation, and with prolonged assay times with human angiotensinogen we were able to detect small quantities of products with $\mathrm{M}+\mathrm{H}^{+}$values of 551, 552, 763 and 1226 . These are likely to be the angiotensinogen fragments RVYI, DRVY, IHPFL and IHPFLVIHN respectively and are probably present due to the action of minor quantities of other venom proteases, emphasising the need for the HIC separation step for full purification of the SVAPs.

Purified SVAP B1 was tested for general protease activity by incubating it at $10 \mu \mathrm{g} / \mathrm{mL}$ with $100 \mu \mathrm{g} / \mathrm{mL}$ alpha or beta casein. GFC-isolated metalloprotease from the same venom batch was used as a positive control. Using the same conditions required to convert $80 \%+$ of angiotensinogen to angiotensin-1, SVAP B1 appeared to have no proteolytic activity towards the two caseins (Fig. 1C). In contrast, the metalloprotease brought about a complete loss of the alpha casein band and a partial digestion of beta casein.

The data presented here confirms the presence in E. ocellatus venom of the aspartic proteases predicted from our transcriptional studies (Wagstaff and Harrison, 2006) and that these possess the postulated renin-like activity. In this previous paper we discussed the possibility that entry of venom aspartic proteases into the vasculature might be expected to induce local hypertension, which may compound SVMP-induced tissue damage, but also systemic hypertension. However, we can find no evidence in the literature for systemic hypertension following $E$. ocellatus envenomation, although such effects may be masked by the complex and rapid pathology associated with viper venom-induced haemorrhage. Indeed, there are very few studies in which hypertension has been demonstrated in the pathology of envenoming by any snake species. In the few cases reporting hypertension symptomology following envenoming by Vipera berus berus (Malina et al., 2008); Proatheris superciliari (Valenta et al., 2008); Bitis nasicornis (Malina and Krecsak, 2008) and Naja haje (Omran and Abdel-Nabi, 1997), hypertension was not attributed to a specific venom component. Where a component of the venom has been indicated as being responsible for hypertension, it was thought to act indirectly e.g. through metalloprotease-induced activation of inactive renin in $B$. arietans (see Morris and Taylor, 1987). Thus, this study is the first in which a venom component has been isolated that may be directly responsible for hypertensive effects of envenomation.

\section{Acknowledgments}

We gratefully acknowledge project grant funding from the Wellcome Trust (072426 awarded to RAH), BBRSC (BB/F012657/ 1 awarded to RAH \& SCW) and Medical Research Council (MR/ L01839X/1 awarded to RAH).

\section{Transparency document}

Transparency document related to this article can be found online at http://dx.doi.org/10.1016/j.toxicon.2017.07.008.

\section{References}

Heukeshoven, J., Dernick, R., 1985. Simplified method for silver staining of proteins in polyacrylamide gels and the mechanism of silver staining. Electrophoresis 6 , $103-112$.

Malina, T., Krecsak, L., 2008. Clinical aspects and consequences of envenoming by active rhinoceros viper (Bitis nasicornis) in Hungary. Swiss Med. Wkly. 138, $85-88$.

Malina, T., Krecsak, L., Warrell, D.A., 2008. Neurotoxicity and hypertension following European adder (Vipera berus berus) bites in Hungary: case report and review. QJM 101, 801-806.

Morris, B.J., Taylor, J.E., 1987. Activation by Puff Adder venom of inactive renin in normal and hypertensive rat plasma. Clin. Exp. Pharmacol. Physiol. 14, 23-31.

Omran, M.A.A., Abdel-Nabi, I.M., 1997. Changes in the arterial blood pressure, heart rate and normal ECG parameters of rat after envenomation with Egyptian cobra (Naja haje) venom. Hum. Exp. Toxicol. 16, 327-333.

Valenta, J., Stach, Z., Fricova, D., Zak, J., Balik, M., 2008. Envenoming by the viperid snake Proatheris superciliaris: a case report. Toxicon 52, 392-394.

Wagstaff, S.C., Harrison, R.A., 2006. Venom gland EST analysis of the saw-scaled viper, Echis ocellatus, reveals novel $\alpha 9 \beta 1$ integrin-binding motifs in venom metalloproteinases and a new group of putative toxins, renin-like aspartic proteases. Gene 377, 21-32. 